\title{
Coexistence of anxiety sensitivity and psychiatric comorbidities in patients with chronic tinnitus
}

This article was published in the following Dove Press journal:

Neuropsychiatric Disease and Treatment

19 February 2015

Number of times this article has been viewed

\author{
Ali İrfan Gül' \\ Mahmut Özkırıș² \\ Reha Aydin² \\ Gülnihal Șimșek ${ }^{3}$ \\ Levent Saydam² \\ 'Department of Psychiatry, \\ ${ }^{2}$ Department of Otolaryngology, Head \\ and Neck Surgery, Bozok University \\ Medical Faculty, ${ }^{3}$ Department of \\ Psychiatry, Government Hospital, \\ Yozgat, Turkey
}

\begin{abstract}
Background: Tinnitus refers to the objective or subjective perception of a series of sounds most frequently described as ringing in the ear or within the head itself. Anxiety and depressive disorders frequently accompany this complaint. In this study, we aimed to investigate the presence of psychiatric symptoms and the degree of anxiety sensitivity in patients with chronic tinnitus.

Methods: Fifty patients with chronic tinnitus who had been followed up for at least 6 months or longer were enrolled in this study. All subjects completed the Anxiety Sensitivity Index-3 (ASI-3), Stait-Trait Anxiety Inventory (STAI), and Symptom Check List-90-Revised (SCL-90-R) questionnaires. Fifty healthy volunteers were given the same tests and a statistical comparison of the psychometric outcome data was done for subjects with and without chronic tinnitus.

Results: Patients with chronic tinnitus demonstrated higher statistically meaningful scores than the healthy group. Comparison between chronic tinnitus group and control group scores showed that patient group has a high rate of statistically significant results than controls; ASI-3, STAI-2, SCL-90-R GSI, SCL-90-R Somatization, SCL-90-R Depression, SCL-90-R Anxiety ( $\mathrm{z}=-8.00, P<0.01)$, SCL-90-R Phobic Anxiety.

Conclusion: Higher scores for anxiety sensitivity and other psychiatric symptoms in patients with chronic tinnitus reflects the prevalence of psychiatric disorders such as depression, anxiety, somatoform disorder, and chronic tinnitus. The finding of more psychiatric comorbidity in patients with chronic tinnitus indicates that planning and follow-up in both otolaryngology and psychiatry is necessary to improve the overall results of treatment.
\end{abstract}

Keywords: anxiety sensitivity, chronic tinnitus, psychiatric disorder

\section{Introduction}

Tinnitus (Latin, meaning "ringing") refers to the perception of sounds without any external stimulus $^{1}$ and is a condition that may be classified as subjective (heard only by the patient) or objective (perceived by both the patient and physician). Objective tinnitus is a rare condition that is usually caused by vascular anomalies or myoclonus of the palatal muscles. Subjective or idiopathic tinnitus is a complaint of an imaginary sound in the head and/ or one or both ears. It is a common symptom in adults and is seen more frequently after 50 years of age. ${ }^{2}$ The condition may result from a dysfunction in the auditory pathway, leading to misperception of a neuroacoustic process. ${ }^{3,4}$ It has been reported that $35 \%-40 \%$ of the adult population in developed countries experience persistent or transient tinnitus at some point in life, with $0.5 \%-2 \%$ of cases involving chronic ( $>6$ months) tinnitus associated with emotional disturbances, deterioration of sleep quality, and social withdrawal. Subjective tinnitus is usually associated with hearing loss, and the condition is more common in men than in women. ${ }^{5,6}$ However, it is not possible to explain the whole picture of tinnitus on the basis of the associated otologic disturbances alone. ${ }^{7}$

Due to the subjective nature of tinnitus, diagnostic evaluation of patients with this condition has always been problematic. As reported earlier, coexistence of various types of cognitive dysfunction result in decreased performance on several tasks that require
Correspondence: Ali Irran Gül Bozok University Medical Faculty, Department of Psychiatry, Adnan Menderes Bulvari 42, Yozgat, Turkey $\mathrm{Tel}+905332184464$

$\mathrm{Fax}+903542125660$

Email gali3366@gmail.com 
memory and attention. ${ }^{8}$ Additionally, electrophysiological studies support dysfunction in brain processes, with dysregulation of event-related potentials and abnormal oculomotor records seen in cases. ${ }^{9}$ Functional imaging-based studies generally suggest that tinnitus has a relationship with maladaptive plasticity occurring in the presence of damage to the auditory system. The dorsolateral prefrontal cortex seems to play an important role in auditory processing, ${ }^{10}$ and the neurophysiological model of tinnitus nowadays is more widely used to describe the phenomenon. ${ }^{11}$

In addition to its known acoustic symptoms, tinnitus is also associated with emotional and cognitive dysfunction. ${ }^{12}$ In many cases, tinnitus is associated with psychological and psychosomatic problems caused by emotional and cognitive dysfunction in addition to the acoustic properties of the condition. A recent study reported that $48 \%-60 \%$ and $45 \%$ of patients with chronic tinnitus developed major depression and anxiety disorder, respectively. ${ }^{13}$ Permanent tinnitus can cause serious deterioration of psychological status and social life and be a source of serious deterioration, assessment of these handicaps sleep disturbances, irritability, psychasthenia, reveals increased relationship with depression and anxiety. ${ }^{14}$ Psychopharmacological and psychiatric approaches have been found to have an important role in the management of patients with tinnitus and comorbid psychiatric disorders, sleep disturbances, and decreased levels of daily activity. ${ }^{15}$ As reported in a recent study, sleep disorders are the second most common comorbidity encountered in patients with chronic tinnitus. ${ }^{16}$ In another study, 39\% of patients with depressive disorder and $45 \%$ of those with anxiety disorder were reported to be at high risk for developing severe tinnitus. ${ }^{17}$

Earlier studies of the individual differences associated with tinnitus were primarily focused on personality characteristics, like neuroticism or coping styles, such as somatic attention. However, several other important psychiatric disorders also increase the risk of developing tinnitus. ${ }^{18}$ Anxiety sensitivity is a condition that should be considered in individuals who are prone to anxiety accompanied by frightening bodily sensations. This concept has been developed in the context of anxiety disorders mostly pointed underlying cognitive vulnerability in the pathogenesis of panic disorder. There has been some research directly investigating the relationship between anxiety sensitivity and tinnitus distress, and a significant correlation was found between the two conditions. ${ }^{18}$

In this study, we investigated the relationship between the presence of psychiatric symptoms and the degree of anxiety sensitivity in patients with chronic tinnitus.

\section{Materials and methods}

This study followed the principles of the Declaration of Helsinki and was approved by the Bozok University Medical Faculty review board. The project design was approved by the noninterventional clinical research ethics committee at our institution (approval number 106). All patients and healthy volunteers were informed about the study.

The study population consisted of 50 patients with a complaint of tinnitus for 6 months or longer selected by a simple random sampling method between March 2013 and July 2013 in the department of otolaryngology at Bozok University School of Medicine. A control group consisting of 50 healthy volunteers was also recruited using the same method. Exclusion criteria were concomitant neuropsychiatric or physical disease, alcohol or drug abuse, and treatment with drugs that may cause tinnitus as a side effect.

The study group included 50 patients with chronic subjective tinnitus, comprising 24 males (48\%) and 26 females $(52 \%)$ of mean age $43.14 \pm 9.45$ years and with a mean tinnitus duration of $12.60 \pm 5.19$ months. The control group comprised 21 males (42\%) and 29 females (58\%) of mean age $41.74 \pm 8.98$ years. There were no statistically significant differences in demographic characteristics between the two groups $(P<0.44, Z=-0.75)$.

A thorough ear, nose, and throat examination was performed in the patients with tinnitus on admission to the otolaryngology clinic. An audiovestibular test battery, including complete audiology and vestibular examinations, was performed in each subject. The Anxiety Sensitivity Index (ASI)-3, State-Trait Anxiety Inventory-1 (STAI-state) and State-Trait Anxiety Inventory-2 (STAI-trait), and Symptom Check List-90-Revised (SCL-90-R) were administered to all patients and healthy volunteers. ASI-3, STAI-trait, and Global Symptom Index (GSI) scores with SCL-90-R somatization, depression, anxiety, and phobic anxiety subscale scores were used in this study.

\section{Anxiety Sensitivity Index-3}

The ASI-3 is a self-rating instrument for measuring anxiety sensitivity and has physical, social, and cognitive dimensions. The ASI-3 can be used in patients with panic disorder, anxiety disorder, or depressive disorder, as well as in healthy volunteers. It consists of 18 items rated on a 5-point Likert-type scale ( 0 , very rare; 4 , very often). The total score consists of the final scores for each item. A cut-off score is not calculated in the Turkish version of the ASI-3. Higher scores indicate increased anxiety sensitivity. The ASI-3 shows high internal consistency and has been found to have 
reasonably good retest reliability $(r=0.64, P<0.001)$. On factor analysis, $61.72 \%$ of the total variance is attributable to physical, cognitive, and social factors. ${ }^{19}$

\section{State-Trait Anxiety Inventory}

The STAI measures transient and ongoing levels of anxiety. Patients over the age of 14 years who understand what they are reading can be evaluated using this test. There are two separate scales, ie, the STAI-state scale and the STAI-trait scale, each having 20 items. The STAI-state scale determines the level of anxiety at a given point in time and the circumstance in which it occurs, and the STAI-trait scale determines the level of anxiety regardless of circumstance. The scale is easy for subjects to self-administer. Both parts of the scale can be applied at the same time, with the STAIstate completed first and STAI-trait completed second, by selection of appropriate choices offered on the STAI-state scale reflecting in terms of degree, thoughts, and behaviors (1, none; 2 , some; 3 , very; 4, completely). When scoring, straight and inverted statements should be considered, and the reverse expressions scoring " 1 " to " 4 ", " 4 " to " 1 " should be converted and collected.

The total score obtained from both subscales on the STAI ranges from 20 to 80 . A high score indicates high levels of anxiety and a low score indicates low levels of anxiety. The scale was developed by Spielberger et $\mathrm{al}^{20}$ and adapted for use in the Turkish population by Öner and Le Compe. ${ }^{21}$

\section{Symptom Check List-90-Revised}

The SCL-90-R is a screening tool that measures psychiatric symptoms and the level of negative response to experienced stress response on a self-rating scale. It can be applied from the age of 17 years in subjects with a minimum of secondary education. There is no time limit for completion of the test, which consists of 90 items rated using a 5-point Likert-type scale.

Each item on the test is answered as "none", "very few", "moderate", "quite a lot", or "very much" and is scored 0,1 , 2,3 , or 4, respectively. Three separate scores are calculated from these scores. The GSI is the overall average score on the scale. An increase in GSI score indicates increased suffering from psychiatric symptoms and is the best indicator of the scale, valued between 0 and 4 scores. A GSI score of $1,0=1.0=1$ is generally recommended as the cut-off score for psychiatric screening. The scale has nine subscales indicating signs of different psychiatric disorders: 1, somatization; 2, obsessive-compulsive symptoms; 3 , interpersonal sensitivity; 4, depression; 5, anxiety; 6, hostility; 7, phobic anxiety; 8 , paranoid thoughts; and 9, psychoticism and additional scales (except these 9 subscales). Subscale scores can have a value between 0 and 4. The SCL-90-R was developed by Derogatis, ${ }^{22}$ and the validity and reliability of the Turkish version was confirmed by Dağ. ${ }^{23}$

\section{Statistical analysis}

The statistical analysis was performed using Statistical Package for the Social Sciences version 17 software (SPSS Inc, Chicago, IL, USA). Descriptive statistics for abnormal distribution variables and ordinal variables were performed by frequency for median, interquartile range. If scores on the scales did not show a normal distribution, the Mann-Whitney $U$-test was used to compare the two groups of subjects. A $P$-value $<0.05$ was considered to be statistically significant. The Spearman test was used to calculate correlation coefficients and statistical significance to show relationships between the scale scores.

\section{Results}

The descriptive statistics were primary performed in the study (Table 1). The scores on the different scales were: ASI-3,

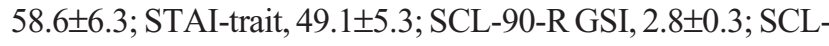
90-R somatization, 2.8 \pm 0.3 ; SCL-90-R depression, 2.9 \pm 0.4 ; SCL-90-R anxiety, 2.8 \pm 0.3 ; and SCL-90-R phobic anxiety, $2.8 \pm 0.4$. The other subscale scores of the SCL-90-R were not evaluated for the purposes of our study.

Correlation analysis was done to investigate whether they are compatible with each other in patients with chronic tinnitus and higher scores (Table 2). Spearman's correlation analysis was performed for the abnormal distribution of some scale scores. ASI-3 scores; SCL-90-R GSI $(\rho=0.401, P<0.01)$, SCL-90-R depression $(\rho=0.416, P<0.01)$, SCL-90-R anxiety $(\rho=0.417, P<0.01)$, SCL-90-R phobic anxiety $(\rho=0.301$, $P<0.05)$ strong correlation between the scores was found in a positive direction. STAI-trait scores and SCL-90-R somatization scores showed a positive correlation $(\rho=0.313, P<0.05)$. A very strong positive correlation was found between SCL90-R GSI and SCL-90-R somatization $(\rho=0.543, P<0.01)$, SCL-90-R depression ( $\rho=0.636, P<0.01)$, SCL-90-R anxiety $(\rho=0.747, P<0.01)$, and SCL-90-R phobic anxiety $(\rho=0.721$, $P<0.01)$.

Finally, scores on all scales were compared between patients with chronic tinnitus and healthy volunteers using the nonparametric Mann-Whitney $U$-test (Table 1) and highly statistically significant results were found for: ASI-3 ( $z=-8.11$, $P<0.001)$, STAI-trait $(z=-4.39, P<0.01)$, SCL-90-R GSI $(z=-8.00, P<0.01)$, SCL-90-R somatization $(z=-8.22$, 
Table I Statistical comparison of chronic tinnitus and control groups

\begin{tabular}{|c|c|c|c|c|c|}
\hline \multirow[b]{2}{*}{ Anxiety Sensitivity Index-3 } & \multirow[b]{2}{*}{ Comparison group } & \multicolumn{2}{|c|}{ Descriptive statistic } & \multirow{2}{*}{$\begin{array}{c}\text { P-value } \\
<0.001\end{array}$} & \multirow{2}{*}{$\frac{\mathbf{Z}^{*}}{-8.11}$} \\
\hline & & Mean & 17.94 & & \\
\hline & & Median & 11.50 & & \\
\hline & & SD & 18.16 & & \\
\hline & Chronic tinnitus & Mean & 58.60 & & \\
\hline & & Median & 62.00 & & \\
\hline & & SD & 6.35 & & \\
\hline \multirow[t]{6}{*}{ State-Trait Anxiety Inventory } & Comparison group & Mean & 43.56 & $<0.001$ & -4.39 \\
\hline & & Median & 44.00 & & \\
\hline & & SD & 6.48 & & \\
\hline & Chronic tinnitus & Mean & 49.16 & & \\
\hline & & Median & 49.00 & & \\
\hline & & SD & 5.37 & & \\
\hline \multirow[t]{6}{*}{ SCL-90-R GSI } & Comparison group & Mean & 0.54 & $<0.001$ & -8.00 \\
\hline & & Median & 0.21 & & \\
\hline & & SD & 0.78 & & \\
\hline & Chronic tinnitus & Mean & 2.84 & & \\
\hline & & Median & 2.85 & & \\
\hline & & SD & 0.32 & & \\
\hline \multirow[t]{6}{*}{ SCL-90-R somatization } & Comparison group & Mean & 0.65 & $<0.001$ & -8.22 \\
\hline & & Median & 0.41 & & \\
\hline & & SD & 0.75 & & \\
\hline & Chronic tinnitus & Mean & 2.88 & & \\
\hline & & Median & 2.91 & & \\
\hline & & SD & 0.31 & & \\
\hline \multirow[t]{6}{*}{ SCL-90-R depression } & Comparison group & Mean & 0.53 & $<0.001$ & -8.19 \\
\hline & & Median & 0.23 & & \\
\hline & & SD & 0.80 & & \\
\hline & Chronic tinnitus & Mean & 2.91 & & \\
\hline & & Median & 2.99 & & \\
\hline & & SD & 0.35 & & \\
\hline \multirow[t]{6}{*}{ SCL-90-R anxiety } & Comparison group & Mean & 0.53 & $<0.001$ & -8.00 \\
\hline & & Median & 0.20 & & \\
\hline & & SD & 0.78 & & \\
\hline & Chronic tinnitus & Mean & 2.88 & & \\
\hline & & Median & 2.90 & & \\
\hline & & SD & 0.37 & & \\
\hline \multirow[t]{6}{*}{ SCL-90-R phobic anxiety } & Comparison group & Mean & 0.42 & $<0.001$ & -8.06 \\
\hline & & Median & 0.14 & & \\
\hline & & SD & 0.78 & & \\
\hline & Chronic tinnitus & Mean & 2.82 & & \\
\hline & & Median & 2.85 & & \\
\hline & & SD & 0.43 & & \\
\hline
\end{tabular}

Note: *Mann-Whitney U-test.

Abbreviations: GSI, Global Symptom Index; SCL-90-R, Symptom Check List-90-Revised; SD, standard deviation.

$P<0.01)$, SCL-90-R depression $(z=-8.19, P<0.01)$, SCL90-R anxiety $(z=-8.00, P<0.01)$, and SCL-90-R phobic anxiety $(z=-8.06, P<0.01)$.

\section{Discussion}

The aims of this study were to investigate anxiety sensitivity in patients with chronic tinnitus and screen them for psychiatric symptoms, and to examine the interrelationship between the data. Statistical comparison of data from patients with chronic tinnitus and healthy volunteers showed significantly higher anxiety sensitivity in the patient group as measured by the ASI-3 $P<0.001, Z=-8.11$. Despite the growing interest in the sensitivity of anxiety in somatic complaints, only a few studies have directly investigated the relationship between anxiety sensitivity and tinnitus. In a study of 146 patients with tinnitus, a significant relationship was found between tinnitus and anxiety sensitivity. ${ }^{18}$ In another study, anxiety and depression in tinnitus patients was found to be common, and psychiatric symptomatology may be important in determining the relationship between anxiety sensitivity and tinnitus complaints, 
Table 2 Correlation between scores on different scales in patients with chronic tinnitus

\begin{tabular}{|c|c|c|c|c|c|c|c|c|}
\hline & & ASI-3 & STAI & $\begin{array}{l}\text { SCL-90-R } \\
\text { GSI }\end{array}$ & $\begin{array}{l}\text { SCL-90-R } \\
\text { somatization }\end{array}$ & $\begin{array}{l}\text { SCL-90-R } \\
\text { depression }\end{array}$ & $\begin{array}{l}\text { SCL-90-R } \\
\text { phobic anxiety }\end{array}$ & $\begin{array}{l}\text { SCL-90-R } \\
\text { anxiety }\end{array}$ \\
\hline \multirow[t]{2}{*}{ ASI-3 } & rho & 1.000 & 0.105 & $0.401 * *$ & 0.036 & $0.416 * *$ & $0.301 *$ & $0.417^{* * *}$ \\
\hline & $P$ & - & 0.470 & 0.004 & 0.806 & 0.003 & 0.034 & 0.003 \\
\hline \multirow[t]{2}{*}{ STAI } & rho & 0.105 & 1.000 & 0.068 & $0.313^{*}$ & 0.149 & 0.145 & 0.195 \\
\hline & $P$ & 0.470 & - & $0.64 I$ & 0.027 & 0.302 & 0.314 & 0.174 \\
\hline \multirow[t]{2}{*}{ SCL-90-R GSI } & rho & $0.40 \mathrm{I} * *$ & 0.068 & 1.000 & $0.543 * *$ & $0.636 * *$ & $0.72 I * *$ & $0.747 * *$ \\
\hline & $P$ & 0.004 & $0.64 I$ & 0.000 & 0.000 & 0.000 & 0.000 & 0.000 \\
\hline \multirow[t]{2}{*}{ SCL-90-R somatization } & rho & 0.036 & $0.313^{*}$ & $0.543 * *$ & 1.000 & $0.550 * *$ & $0.586 * *$ & $0.439 * *$ \\
\hline & $P$ & 0.806 & 0.027 & 0.000 & 0.000 & 0.000 & 0.000 & 0.001 \\
\hline \multirow[t]{2}{*}{ SCL-90-R depression } & rho & $0.416 * *$ & 0.149 & $0.636 * *$ & $0.550 * *$ & 1.000 & $0.513^{* *}$ & $0.689 * *$ \\
\hline & $P$ & 0.003 & 0.302 & 0.000 & 0.000 & 0.000 & 0.000 & 0.000 \\
\hline \multirow[t]{2}{*}{ SCL-90-R phobic anxiety } & rho & $0.301 *$ & 0.145 & $0.721^{* *}$ & $0.586 * *$ & $0.513 * *$ & 1.000 & $0.591 * *$ \\
\hline & $P$ & 0.034 & 0.314 & 0.000 & 0.000 & 0.000 & 0.000 & 0.000 \\
\hline \multirow[t]{2}{*}{ SCL-90-R anxiety } & rho & $0.417 * *$ & 0.195 & $0.747^{* *} *$ & $0.439 * *$ & $0.689 * *$ & $0.591 * *$ & 1.000 \\
\hline & $P$ & 0.003 & 0.174 & 0.000 & 0.001 & 0.000 & 0.000 & - \\
\hline
\end{tabular}

Notes: **Correlation is significant at the 0.01 level (two-tailed); *correlation is significant at the 0.05 level (2-tailed).

Abbreviations: ASI-3, Anxiety Sensitivity Index-3; GSI, Global Symptom Index; SCL-90-R, Symptom Check List-90-Revised; STAI, Stait-Trait Anxiety Inventory.

in parallel to our results. These studies suggest that anxiety sensitivity is a very important mediating variable affecting the symptom of tinnitus complaint. ${ }^{24}$ Anxiety sensitivity is a most important vulnerability factor in the development of psychopathology generally. ${ }^{25}$ Anxiety sensitivity is seen as cognitive vulnerability causing panic disorder and related symptoms historically. In studies comparing patients with panic disorder with healthy normal volunteers, anxiety sensitivity is consistently found at higher levels in patients with panic disorder. Also, anxiety sensitivity is being related spontaneous panic attacks. ${ }^{24,26}$

One of the important results of our study is that STAItrait scores were noticeably higher in patients than in controls (Table 1). This finding correlates significantly and positively with scores on the somatization subscale of the SCL-90-R showing somatic anxiety at high levels in patients with tinnitus. It has been debated as to whether there is a strong relationship between perception of tinnitus and anxiety. ${ }^{27}$ Anxiety does not produce tinnitus by itself but it can strongly kindle the perception of it. Anxiety is considered to be one of the key factors in the psychological model of the tolerance to cope with tinnitus or treatment of tinnitus, and is also thought to be a causal factor in the intensity of tinnitus experienced by patients. ${ }^{28}$

In our study, the three scales that we used was SCL-90-R as shown by the scores that indicate distress caused by psychiatric symptoms were found to be considerably higher in the study group than control group in this scales. Somatization, depression, anxiety, and phobic anxiety subgroup scale scores on the SCL-90-R were found to be relatively higher in the tinnitus group $(P<0.001)$. Ninety-three percent of patients who suffered from tinnitus reported psychiatric symptoms. Mental disorders, including depression, dysthymia, insomnia, loss of concentration, somatoform disorders, anxiety, and obsessive compulsive disorder have been reported to occur in up to $46 \%$ of patients. ${ }^{28}$ Some groups ${ }^{29}$ have found an important relationship between mental health and tinnitus or sensitivity to noise. Lifetime rates of major depression in tinnitus patients were higher than in controls (61\% versus 21\%). ${ }^{29}$ Other researchers found that patients with tinnitus who also suffered from anxiety or depression had higher scores on all the SCL-90-R subgroup scales, ${ }^{29,30}$ which is compatible with our study. The relationship between severity of tinnitus and depression indicates that different personality traits and coping skills could be effective in defining the symptoms. Increased tinnitus levels may also lead to increased anxiety levels and at the same time anxiety reduces the tolerance to tinnitus, creating a vicious circle. ${ }^{31}$ It has been reported that emotional factors such as depression and anxiety disorder are strong indicators of a poor prognosis in patients with tinnitus. ${ }^{32}$ Different medical and psychotherapeutic treatments have been developed and tested to reduce the severity of tinnitus and related symptoms. Even though antidepressants do not directly cure tinnitus, they do cure comorbid anxiety and depression, and are believed to be useful in patients with tinnitus. ${ }^{33}$ Cognitive behavioral treatment modalities in particular have been demonstrated to ameliorate the problems caused by tinnitus. ${ }^{34-38}$

In conclusion, patients with chronic tinnitus are very prone to developing psychiatric conditions, such as anxiety sensitivity, anxiety, and depression, indicating that multidisciplinary approaches are mandatory in the management of these patients.

\section{Disclosure}

The authors report no conflicts of interest in this work. 


\section{References}

1. Malakouti S, Mahmoudian M, Alifattahi N, Salehi M. Comorbidity of chronic tinnitus and mental disorders. Int Tinnitus J. 2011;16(2): $118-122$.

2. Hebert S, Lupien SJ. The sound of stress: blunted cortisol reactivity to psychosocial stress in tinnitus sufferers. Neurosci Lett. 2007;411(2): 138-142.

3. Schaaf H, Eichenberg C, Kastellis G, Hesse G. Treatment of tinnitus needs a combined neurootological and psychosomatic approach. Otolaryngol Pol. 2010;64(2):78-82.

4. Møller AR. Neural plasticity in tinnitus. Prog Brain Res. 2006;157: 365-372.

5. Salviati M, Macri F, Terlizzi S, et al. The tinnitus handicap inventory (THI) as a screening test for psychiatric comorbidity in patients with tinnitus. Psychosomatics. 2013;54(3):248-256.

6. Langenbach M, Olderog M, Michel O, Albus C, Köhle K. Psychosocial and personality predictors of tinnitus-related distress. Gen Hosp Psychiatry. 2005;27(1):73-77.

7. Axelsson A, Ringdahl A. Tinnitus - a study of its prevalence and characteristics. Br J Audiol. 1989;23(1):53-62.

8. Rossiter S, Stevens C, Walker G. Tinnitus and its effect on working memory and attention. $J$ Speech Lang Hear Res. 2006;49(1):150-160.

9. Gabr TA, El-Hay MA, Badawy A. Electrophysiological and psychological studies in tinnitus. Auris Nasus Larynx. 2011;38(6):678-683.

10. Faber M, Vanneste S, Fregni F, De Ridder D. Top down prefrontal affective modulation of tinnitus with multiple sessions of tDCS of dorsolateral prefrontal cortex. Brain Stimul. 2012;5(4):492-498.

11. Lopez-Gonzalez MA, Moliner-Peiro F, Alfaro-Garcia J, EstebanOrtega F. Sulpiride plus hydroxyzine decrease tinnitus perception. Auris Nasus Larynx. 2007;34(1):23-27.

12. Milerová J, Anders M, Dvořák T, Sand PG, Königer S, Langguth B. The influence of psychological factors on tinnitus severity. Gen Hosp Psychiatry. 2013;35(4):412-416.

13. Belli H, Oktay MF, Ural C. Psychopathological dimensions of tinnitus and psychopharmacologic approaches in its treatment. Gen Hosp Psychiatry. 2012;34(3):282-289.

14. Job A, Cian C, Esquivie D, et al. Moderate variations of mood/emotional states related to alterations in cochlear otoacoustic emissions and tinnitus onset in young normal hearing subjects exposed to gun impulse noise. Hear Res. 2004;193(1-2):31-38.

15. Andersson G, Kaldo-Sandström V, Ström L, Strömgren T. Internet administration of the Hospital Anxiety and Depression Scale in a sample of tinnitus patients. J Psychosom Res. 2003;55(3):259-262.

16. Wallhausser-Franke E, Schredl M, Delb W. Tinnitus and insomnia: is hyperarousal the common denominator? Sleep Med Rev. 2013;17(1):65-74.

17. Zöger S, Svedlund J, Holgers K-M. Relationship between tinnitus severity and psychiatric disorders. Psychosomatics. 2006;47(4):282-288.

18. Hesser H, Andersson G. The role of anxiety sensitivity and behavioral avoidance in tinnitus disability. Int J Audiol. 2009;48(5):295-299.

19. Mantar A, Yemez B, Alkın T. [The validity and reliability of the Turkish version of the Anxiety Sensitivity Index-3]. Turk Psikiyatri Derg. 2010;21(3):225-234. Turkish.

20. Spielberger CD, Gorsuch RL, Lushene RE. Manuel for State-Trait Anxiety Inventory. California: Consulting Psychologist Press. 1970;1-23.

21. Öner N, Le Compe A. State-Trait Anxiety Inventory Hand Book. Istanbul, Turkey: Boğaziçi University; 1985.
22. Derogatis LR. SCL-90: Administration, Scoring and Procedure Manual-I for the R (Revised) Version. Baltimore, MD, USA: Johns Hopkins University School of Medicine; 1977.

23. Dağ İ. Belirti Tarama Listesi (SCL-90-R) nin üniversite öğrencileri için güvenilirliği ve geçerliliği [Symptom check list (SCL 90R) Reliability and Validity study for University Students]. Turk Psikiyatri Derg. 1991;2(1):5-12. Turkish.

24. Andersson G, Vretblad P. Anxiety sensitivity in patients with chronic tinnitus. Scand J Behav Ther. 2000;29(2):57-64.

25. Zinbarg RW, Brown TA, Barlow DH, Rapee RM. Anxiety sensitivity, panic and depressed mood: are analysis teasing apart the contributions of the two levels in the hierarchical structure of the Anxiety Sensitivity Index. J Abnorm Psychol. 2001;110(3):372-377.

26. Tull MT, Gratz KL. Further examination of the relationship between anxiety sensitivity and depression: the mediating role of experiential avoidance and difficulties engaging in goal-directed behavior when distressed. J Anxiety Disord. 2008;22(2):199-210.

27. Ooms E, Vanheule S, Meganck R, Vinck B, Watelet J-B, Dhooge I. Tinnitus severity and its association with cognitive and somatic anxiety: a critical study. Eur Arch Otorhinolaryngol. 2012;269(11):2327-2333.

28. Zirke N, Seydel C, Arsoy D, et al. Analysis of mental disorders in tinnitus patients performed with composite international diagnostic interview. Qual Life Res. 2013;22(8):2095-2104.

29. Harrop-Griffiths J, Katon W, Dobie R, Sakai C, Russo J. Chronic tinnitus: association with psychiatric diagnoses. J Psychosom Res. 1987;31(5):613-621.

30. Falkenberg ES, Wie OB. Anxiety and depression in tinnitus patients: 5-year follow-up assessment after completion of habituation therapy. Int J Otolaryngol. 2012;2012:375-460.

31. Krog NH, Engdahl B, Tambs K. The association between tinnitus and mental health in a general population sample: results from the HUNT study. J Psychosom Res. 2010;69(3):289-298.

32. Zöger S, Svedlund J, Holgers KM. Psychiatric disorders in tinnitus patients without severe hearing impairments: 24 month follow-up of patients at an audiological clinic. Audiology. 2001;40(3):133-140.

33. Robinson SK, Viirre ES, Stein MB. Antidepressant therapy in tinnitus. Hear Res. 2007;226(1-2):221-231.

34. Weise C, Kleinstauber M, Hesser H, Westin VZ, Andersson G. Acceptance of tinnitus: validation of the Tinnitus Acceptance Questionnaire. Cogn Behav Ther. 2013;42(2):100-115.

35. Cima RF, Maes IH, Joore MA, et al. Specialised treatment based on cognitive behaviour therapy versus usual care for tinnitus: a randomised controlled trial. Lancet. 2012;379(9830):1951-1959.

36. Fornaro M, Martino M. Tinnitus psychopharmacology: a comprehensive review of its pathomechanisms and management. Neuropsychiatr Dis Treat. 2010;6:209-218.

37. Kröner-Herwig B, Frenzel A, Fritsche G, Schilkowsky G, Esser G. The management of chronic tinnitus: comparison of an outpatient cognitive-behavioral group training to minimal-contact interventions. J Psychosom Res. 2003;54(4):381-389.

38. Hesser H, Weise C, Westin VZ, Andersson G. A systematic review and meta-analysis of randomized controlled trials of cognitive-behavioral therapy for tinnitus distress. Clin Psychol Rev. 2011;31(4):545-553.
Neuropsychiatric Disease and Treatment

\section{Publish your work in this journal}

Neuropsychiatric Disease and Treatment is an international, peerreviewed journal of clinical therapeutics and pharmacology focusing on concise rapid reporting of clinical or pre-clinical studies on a range of neuropsychiatric and neurological disorders. This journal is indexed on PubMed Central, the 'PsycINFO' database and CAS,

\section{Dovepress}

and is the official journal of The International Neuropsychiatric Association (INA). The manuscript management system is completely online and includes a very quick and fair peer-review system, which is all easy to use. Visit http://www.dovepress.com/testimonials.php to read real quotes from published authors. 